\title{
One-Vs-All Training of Prototype Classifier for Pattern Classification and Retrieval
}

\author{
Cheng-Lin Liu \\ National Laboratory of Pattern Recognition, \\ Institute of Automation, Chinese Academy of Sciences \\ 95 Zhongguancun East Road, Beijing 100190, P.R. China \\ liucl@nlpr.ia.ac.cn
}

\begin{abstract}
Prototype classifiers trained with multi-class classification objective are inferior in pattern retrieval and outlier rejection. To improve the binary classification (detection, verification, retrieval, outlier rejection) performance of prototype classifiers, we propose a onevs-all training method, which enriches each prototype as a binary discriminant function with a local threshold, and optimizes both the prototype vectors and the thresholds on training data using a binary classification objective, the cross-entropy $(C E)$. Experimental results on two OCR datasets show that prototype classifiers trained by the one-vs-all method is superior in both multi-class classification and binary classification.
\end{abstract}

\section{Introduction}

The nearest prototype classifier (NPC) classifies the test (query) pattern to the class of the nearest prototype. High classification accuracies can be achieved by the NPC with a small number of prototypes when properly selected. The so-far proposed prototype learning methods can be grouped into ones based on heuristic adjustment (such as learning vector quantization (LVQ) [1]) and ones optimizing an objective function (such as $[2,3,4,5,6])$. These methods, optimizing a multi-class objective in training, do not consider outliers, which exist prevalently in applications of image recognition and document analysis, such as character string recognition [7], keyword retrieval [8] and transcript mapping [9].

For a prototype classifier trained with multi-class objective, the prototypes may move toward the regions of outliers in the feature space, and thus fail to reject the outliers in these regions. Regularizing the objective for attracting prototypes toward the distributed regions of defined classes helps improve outlier rejection [7], but does not suffice. The method of [10] learns a rejection threshold together with the prototypes in a multi-class formulation (class probabilities computed by soft-max), but entails outlier samples to learn a proper threshold.

Aiming to improve the rejection ability of prototype classifier while preserving the multi-class classification accuracy, this paper proposes a one-vs-all training method. The proposed method enriches each prototype with a local threshold, and optimizes the prototypes and thresholds under a binary criterion, the crossentropy (CE). Since the binary classifier of each class is trained to separate the class from the others, it resists outliers as well. Each binary classifier has prototypes for the positive class only. Our method can be viewed as a prototype specific metric learning, similar to local distance metric [11] and category specific metric [12], and can be extended to non-Euclidean metrics.

Our experimental results on two OCR datasets show that by one-vs-all training, the rejection performance of prototype classifiers is largely improved while the classification accuracy is comparable with those trained by multi-class objective.

\section{Nearest Prototype Classifier}

For classifying a $d$-dimensional pattern $\mathbf{x}$ into $M$ pre-defined classes $\left\{\omega_{1}, \ldots, \omega_{M}\right\}$, the prototype classifier has $n_{i}$ prototypes $\left\{\mathbf{m}_{i j}, j=1, \ldots, n_{i}\right\}$ for each class $\omega_{i}, i=1, \ldots, M$. The test (query) pattern $\mathbf{x}$ is classified to the class of the nearest prototype:

$$
\arg \min _{i}\left\{\min _{j}\left\|\mathbf{x}-\mathbf{m}_{i j}\right\|^{2}\right\} \text {. }
$$

Usually, the prototypes of each class are initialized as the cluster centers of classwise training data. Then in prototype learning, the prototypes of all classes are iteratively adjusted under a classification objective. 
For comparison with our proposed prototype learning method, we select some representative previous algorithms, which are outlined as follows.

Given a training dataset $\left\{\left(\mathbf{x}^{n}, c^{n}\right) \mid n=1, \ldots, N\right\}$ ( $c^{n}$ is the class label of $\mathbf{x}^{n}$ ), the prototypes $\Theta=$ $\left\{\mathbf{m}_{i j} \mid i=1, \ldots, M, j=1, \ldots, n_{k}\right\}$ are updated to minimize the empirical loss

$$
\min _{\Theta} L_{0}=\frac{1}{N} \sum_{n=1}^{N} l_{c}\left(\mathbf{x}^{n}, \Theta\right),
$$

where $l_{c}\left(\mathbf{x}^{n}, \Theta\right)$ is the misclassification loss on $\mathbf{x}^{n}$.

The MCE algorithm [2] defines the loss as a smoothed probability of misclassification:

$$
l_{c}(\mathbf{x}, \Theta)=\sigma[\xi \mu(\mathbf{x})],
$$

where

$$
\mu(\mathbf{x})=\left\|\mathbf{x}-\mathbf{m}_{c i}\right\|^{2}-\left\|\mathbf{x}-\mathbf{m}_{r j}\right\|^{2} .
$$

$\sigma(\cdot)$ is the sigmoid function, $\xi$ is a scaling parameter, $\mathbf{m}_{c i}$ is the nearest prototype of $\mathbf{x}$ from the genuine class $\omega_{c}$ and $\mathbf{m}_{r j}$ is the nearest from incorrect classes.

The LOGM algorithm [6] optimizes a log-likelihood loss of hypothesis margin:

$$
l_{c}(\mathbf{x}, \Theta)=-\log \sigma\left[\xi\left(\left\|\mathbf{x}-\mathbf{m}_{r j}\right\|^{2}-\left\|\mathbf{x}-\mathbf{m}_{c i}\right\|^{2}\right)\right] .
$$

The log-likelihood loss is a convex function and thus leads to better convergence in gradient learning.

The RSLVQ algorithm [5] optimizes a log-likelihood loss under a framework of Gaussian mixture density:

$$
l_{c}(\mathbf{x}, \Theta)=-\log \frac{\sum_{j=1}^{n_{c}} \exp \left(-\xi\left\|\mathbf{x}-\mathbf{m}_{c j}\right\|^{2}\right)}{\sum_{i=1}^{M} \sum_{j=1}^{n_{i}} \exp \left(-\xi\left\|\mathbf{x}-\mathbf{m}_{i j}\right\|^{2}\right)},
$$

By minimizing this loss, the RSLVQ algorithm updates all the prototypes on a training pattern in gradient descent, unlike the MCE and LOGM that update only two prototypes on a training pattern.

A regularization term can be added to the objective of prototype learning to attract the prototypes toward the distributed regions of pre-defined classes [7]:

$$
L_{1}=\frac{1}{N} \sum_{n=1}^{N}\left[l_{c}\left(\mathbf{x}^{n}, \Theta\right)+\alpha\left\|\mathbf{x}^{n}-\mathbf{m}_{c i}\right\|^{2}\right] .
$$

\section{One-Vs-All Training Method}

The prototype classifier has not been trained by onevs-all strategy because it is inherently a multi-class classifier, unlike the support vector machine (SVM) and AdaBoost that are inherently binary classifiers. To train the prototype classifier using one-vs-all strategy, we reformulate the classification rule by adding a threshold to each prototype:

$$
\arg \min _{i}\left\{\min _{j}\left(\left\|\mathbf{x}-\mathbf{m}_{i j}\right\|^{2}-\tau_{i j}\right)\right\} .
$$

We can view $f_{i j}(\mathbf{x})=-\left(\left\|\mathbf{x}-\mathbf{m}_{i j}\right\|^{2}-\tau_{i j}\right)$ as a binary discriminant function. The discriminant function of a class is

$$
f_{i}(\mathbf{x})=\max _{j} f_{i j}(\mathbf{x})=-\min _{j}\left(\left\|\mathbf{x}-\mathbf{m}_{i j}\right\|^{2}-\tau_{i j}\right) .
$$

$f_{i}(\mathbf{x})$ classifies $\mathbf{x}$ as belong to $\omega_{i}$ when $f_{i}(\mathbf{x})>0$ and not belong to $\omega_{i}$ when $f_{i}(\mathbf{x})<0$. The probability of classifying to $\omega_{i}$ is

$$
p_{i}(\mathbf{x})=P\left(\omega_{i} \mid \mathbf{x}\right)=\sigma\left[\xi f_{i}(\mathbf{x})\right] .
$$

For training on a dataset $\left\{\left(\mathbf{x}^{n}, c^{n}\right) \mid n=1, \ldots, N\right\}$, define $M$ binary classification problems: $\omega_{i}$ versus non$\omega_{i}, i=1, \ldots, M$. The multi-class label $c^{n}$ is transformed to $M$ binary labels $y_{i}^{n}=\delta\left(c^{n}, i\right) \in\{0,1\}$,

$i=1, \ldots, M$. By maximizing the log-likelihood $\log \prod_{i}^{M} p_{i}^{y_{i}^{n}}\left(1-p_{i}\right)^{1-y_{i}^{n}}$, the cross-entropy (CE) loss is obtained:

$$
\begin{aligned}
& \min _{\Theta} C E \\
& =-\sum_{n=1}^{N} \sum_{i=1}^{M}\left[y_{i}^{n} \log p_{i}+\left(1-y_{i}^{n}\right) \log \left(1-p_{i}\right)\right] \\
& =-\sum_{i=1}^{M} \sum_{n=1}^{N}\left[y_{i}^{n} \log p_{i}+\left(1-y_{i}^{n}\right) \log \left(1-p_{i}\right)\right] .
\end{aligned}
$$

The summation over $M$ classes implies that the training problem can be decomposed into $M$ binary problems. This is similar to the training of multilayer neural networks, where both the $\mathrm{CE}$ and the squared loss can be adopted. Using squared loss may give similar performance to $\mathrm{CE}$, but because the $\mathrm{CE}$ is a convex function of $f_{i}(\mathbf{x})$, better convergence in training can be expected.

Similar to (7), the CE function can be added a regularization term:

$$
\begin{aligned}
& C E_{1}= \\
& \begin{aligned}
-\sum_{n=1}^{N} & \left\{\sum_{i=1}^{M}\left[y_{i}^{n} \log p_{i}+\left(1-y_{i}^{n}\right) \log \left(1-p_{i}\right)\right]\right. \\
& \left.-\alpha\left\|\mathbf{x}^{n}-\mathbf{m}_{c l}\right\|^{2}\right\}
\end{aligned}
\end{aligned}
$$

where $\mathbf{m}_{c l}$ is the nearest prototype of $\mathbf{x}^{n}$ from the genuine class $c^{n}$.

To minimize the objective of (12) by stochastic gradient descent, the training samples are fed to the prototype classifier iteratively. On a training sample $\mathrm{x}^{n}$ at time $t$, the nearest prototype from each class is retrieved 
and is updated with its threshold:

$$
\begin{aligned}
\mathbf{m}_{i l}(t+1)= & \mathbf{m}_{i l}(t)+2 \eta_{1}(t)\left[\xi\left(1-p_{i}\right)+\alpha\right]\left(\mathbf{x}^{n}-\mathbf{m}_{i l}\right) \\
& i=c^{n} \\
\mathbf{m}_{i l}(t+1)= & \mathbf{m}_{i l}(t)-2 \eta_{1}(t) \xi p_{i}\left(\mathbf{x}^{n}-\mathbf{m}_{i l}\right) \\
& i \neq c^{n} \\
\tau_{i l}(t+1)= & \tau_{i l}(t)+\eta_{2}(t) \xi\left[y_{i}^{n}\left(1-p_{i}\right)-\left(1-y_{i}^{n}\right) p_{i}\right] \\
& i=1, \ldots, M
\end{aligned}
$$

where $\mathbf{m}_{i l}$ is the nearest prototype to $\mathbf{x}^{n}$ from class $\omega_{i}$.

After initializing prototypes by $\mathrm{k}$-means clustering of classwise data, the updating proceeds for a number of cycles of training samples, with the learning steps $\eta_{1}$ and $\eta_{2}$ shrinking gradually. For good convergence, the scaling parameter $\xi$ is selected to be inversely proportional to the average within-cluster sample-to-center distance $(v a r)$. The thresholds $\tau_{i j}$ are empirically initialized as $2 v a r$. The initial learning step $\eta_{1}(0)$ is inversely proportional to $\xi$, and the ratio of two learning steps $\eta_{2} / \eta_{1}$ is set equal to var. The regularization coefficient is set as $\alpha=\alpha_{0} / v a r$, with $\alpha_{0} \in[0,1]$.

The one-vs-all training method can be easily applied to training with outlier samples, for which the binary labels $y_{i}^{n}=0, i=1, \ldots, M$. This can further improve the outlier rejection ability of prototype classifier.

\section{Experimental Results}

For evaluation, we experimented on two OCR datasets, USPS handwritten digits and UCI Letter Recognition dataset. The USPS dataset contains digit images of normalized size $16 \times 16$, with 7,291 samples for training and 2,007 samples for testing. The Letter dataset contains 16,000 samples for training and 4,000 samples for testing, each represented in 16-dimensional features. We trained prototype classifiers using four algorithms: MCE, LOGM, RSLVQ, and the proposed one-vs-all cross-entropy (OVACE) method. After training, the multi-class classification accuracy and the binary one-vs-all classification measures are given.

The binary classification can be viewed as a pattern retrieval or verification task: a test sample is compared with each defined class to judge whether it belongs to the class or not. The discriminant function in (9) $\left(\tau_{i j}=\right.$ 0 for prototype classifiers trained by MCE, LOGM and RSLVQ) is compared with an artificial threshold $\tau_{0}$ : if $f_{i}(\mathbf{x})>\tau_{0}, \mathbf{x}$ is judged to belong to $\omega_{i}\left(\omega_{i}\right.$ is retrieved $)$, otherwise $\mathbf{x}$ does not belong to $\omega_{i}$. For a sample $\mathbf{x}$ from class $\omega_{c}$, the decision of belonging to $\omega_{i}$ results in a true positive if $i=c$, otherwise a false positive. The rates of true positive and false positive with variable values of $\tau_{0}$ give the ROC curve.

Even though we did not evaluate on real outlier samples, the results of retrieval are meaningful because a sample $\mathbf{x}$ from class $\omega_{c}$ is a negative sample for all the other classes $\omega_{i}(i \neq c)$. So, a low rate of false positive indicates that the classifier can reject outliers well.

In our experiments, we set equal number of $n$ prototypes for each class: $n=6,8,10$ for USPS and $n=20,30,40$ for Letter. The regularization coefficient $\alpha_{0}$ was set to 0.05 for all the methods. The multiclass classification error rates on the test samples of two datasets are shown in Table 1 and Table 2, respectively, and the ROC curves of pattern retrieval are shown in Fig. 1 and Fig. 2, respectively.

We have also set classwise thresholds to the classifiers trained by MCE, LOGM and RSLVQ, for these methods had no local thresholds in prototype learning. This was done by confidence transformation (CT) using logistic regression [13]: the discriminant function of each class $f_{i}(\mathbf{x})$ is mapped to binary probability by $\sigma\left[a_{i} f_{i}(\mathbf{x})+b_{i}\right]$, with parameters $\left(a_{i}, b_{i}\right)$ estimated by cross-entropy minimization on the training dataset. The class discriminant function is then replaced by $a_{i} f_{i}(\mathbf{x})+b_{i}$. The classification error rates on two datasets after CT are shown in Table 1 and Table 2, respectively, and the ROC curves are shown in Fig. 1 and Fig. 2, respectively.

Table 1. Classification error rates (\%) on USPS dataset, before and after confidence transformation (CT).

\begin{tabular}{|c|ccc|ccc|}
\hline & \multicolumn{3}{|c|}{ Before CT } & \multicolumn{3}{c|}{ After CT } \\
\hline$n$ & 6 & 8 & 10 & 6 & 8 & 10 \\
\hline MCE & 5.63 & $\mathbf{5 . 4 3}$ & 5.68 & 6.18 & $\mathbf{6 . 0 8}$ & $\mathbf{6 . 0 8}$ \\
LOGM & 5.58 & $\mathbf{5 . 5 3}$ & 6.03 & $\mathbf{6 . 0 8}$ & 6.38 & 6.33 \\
RSLVQ & $\mathbf{5 . 5 8}$ & 5.63 & 5.88 & $\mathbf{5 . 7 3}$ & 5.88 & 5.78 \\
OVACE & 6.58 & $\mathbf{5 . 8 3}$ & $\mathbf{5 . 8 3}$ & 6.43 & $\mathbf{5 . 6 3}$ & 5.73 \\
\hline
\end{tabular}

Table 2. Classification error rates (\%) on Letter dataset, before and after confidence transformation (CT).

\begin{tabular}{|c|ccc|ccc|}
\hline & \multicolumn{3}{|c|}{ Before CT } & \multicolumn{3}{c|}{ After CT } \\
\hline$n$ & 20 & 30 & 40 & 20 & 30 & 40 \\
\hline MCE & $\mathbf{5 . 0 8}$ & 5.25 & 5.10 & 6.15 & 5.97 & $\mathbf{5 . 7 5}$ \\
LOGM & 4.92 & 5.03 & $\mathbf{4 . 7 2}$ & 6.17 & 6.22 & $\mathbf{5 . 5 5}$ \\
RSLVQ & $\mathbf{3 . 9 7}$ & 4.80 & 4.62 & $\mathbf{5 . 0 5}$ & 5.65 & 5.40 \\
OVACE & 6.30 & 5.47 & $\mathbf{4 . 6 7}$ & 6.33 & 5.37 & $\mathbf{4 . 7 2}$ \\
\hline
\end{tabular}

The results in Table 1 and Table 2 show that the multi-class classification accuracy of the one-vs-all training method is slightly inferior or comparable to 

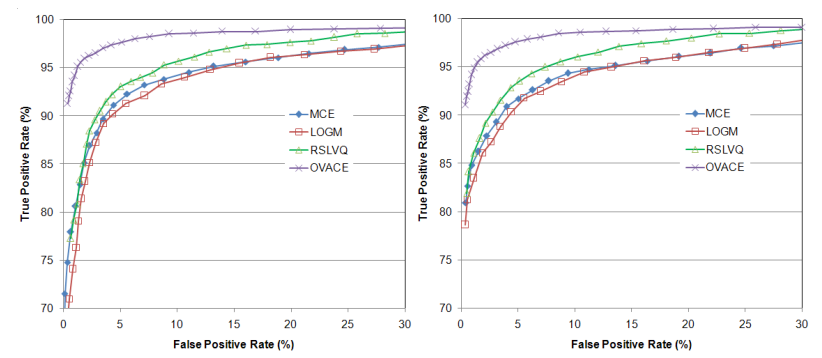

Figure 1. ROC of pattern retrieval on USPS dataset ( $n=10)$, before (left) and after confidence transformation (right).
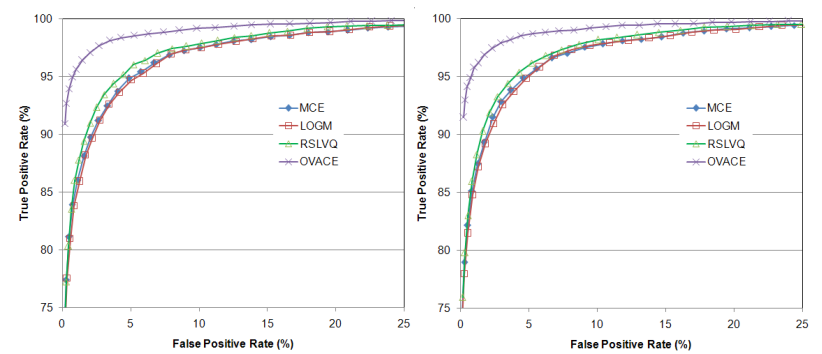

Figure 2. ROC of pattern retrieval on Letter dataset ( $n=40$ ), before (left) and after confidence transformation (right).

those of MCE, LOGM and RSLVQ. However, after confidence transformation (CT), the classification accuracies of prototype classifiers trained by MCE, LOGM and RSLVQ were lowered, while the accuracy of onevs-all training was hardly changed by CT. This is because the $\mathrm{CT}$ also optimizes a one-vs-all objective.

The ROC curves in Fig. 1 and Fig. 2 show that onevs-all training yields superior performance of pattern retrieval, and the prototype classifiers trained with multiclass objective perform very poor in pattern retrieval. For the classifiers trained by MCE, LOGM and RSLVQ, confidence transformation slightly improves the pattern retrieval performance because classwise thresholds are learned, but the retrieval performance is still inferior to that of one-vs-all prototype learning.

\section{Conclusion}

This paper proposes a one-vs-all training method for prototype classifiers by optimizing a binary classification objective, unlike the previous prototype learning methods that optimize a multi-class objective. Our experimental results on OCR datasets demonstrate the superior classification and pattern retrieval performance of one-vs-all training. In our future work, we will evaluate prototype classifiers trained by the one-vs-all method on real outlier (out-of-class) samples, and apply such trained classifiers to character string recognition, keyword retrieval and transcript mapping.

\section{Acknowledgements}

This work was supported by the National Natural Science Foundation of China (NSFC) under grants no.60775004, no.60825301 and no.60933010.

\section{References}

[1] T. Kohonen, The self-organizing map, Proc. IEEE, 78(9): 1464-1480, 1990.

[2] B.-H. Juang, S. Katagiri, Discriminative learning for minimum error classification, IEEE Trans. Signal Processing, 40(12): 3043-3054, 1992.

[3] A. Sato and K. Yamada, A formulation of learning vector quantization using a new misclassification measure, Proc. 14th ICPR, Brisbane, 1998, Vol.I, pp.322-325.

[4] C.-L. Liu, M. Nakagawa, Evaluation of prototype learning algorithms for nearest neighbor classifier in application to handwritten character recognition, Pattern Recognition, 34(3): 601-615, 2001.

[5] S. Seo, K. Obermayer, Soft learning vector quantization, Neural Computation, 15(7): 1589-1604, 2003.

[6] X.-B. Jin, C.-L. Liu, X. Hou, Regularized margin-based conditional log-likelihood loss for prototype learning, Pattern Recognition, 43(7): 2428-2438, 2010.

[7] C.-L. Liu, H. Sako, H. Fujisawa, Effects of classifier structures and training regimes on integrated segmentation and recognition of handwritten numeral strings, IEEE Trans. PAMI, 26(11): 1395-1407, 2004.

[8] H. Cao, A. Bhardwaj, V. Govindaraju, A probabilistic method for keyword retrieval in handwritten document images, Pattern Recognition, 42(12): 3374-3382, 2009.

[9] F. Yin, Q.-F. Wang, C.-L. Liu, A tool for groundtruthing text lines and characters in off-line handwritten Chinese documents, Proc. 10th ICDAR, Barcelona, Spain, 2009.

[10] K. Urahama, Y. Furukawa, Gradient descent learning of nearest neighbor classifiers with outlier rejection, Pattern Recognition, 28(5): 761-768, 1995.

[11] A. Frome, Y. Singer, F. Sha, J. Malik, Learning globally-consistent local distance functions for shapebased image retrieval and classification, Proc. 11th ICCV, Rio de Jeneiro, Brazil, 2007.

[12] B. Babenko, S. Branson, S. Belongie, Similarity metrics for cateforization: from monolithic to category specific, Proc. 12th ICCV, Kyoto, Japan, 2009.

[13] L. Gillick, Y. Ito, J. Young, A probabilistic approach to confidence estimation and evaluation, Proc. ICASSP'97, Munich, Germany, Vol.2, pp.879-882. 